\title{
Contribution to determining the antioxidant capacity of melatonin in orodispersible tablets - comparison with reference antioxidants
}

\author{
Herminia Muñoz ${ }^{1}$, Sergio García², Adolfina Ruiz ${ }^{1}$
}

\author{
${ }^{1}$ Pharmacy and Pharmaceutical Technology Department, School of Pharmacy, \\ University of Granada, Granada, Spain \\ ${ }^{2}$ Zoology Department, School of Science, University of Granada, Granada, Spain
}

Submitted: 5 June 2017

Accepted: 10 October 2017

Arch Med Sci 2020; 16 (4): 871-877

DOI: https://doi.org/10.5114/aoms.2020.94106

Copyright $\odot 2020$ Termedia \& Banach

\section{Abstract}

Introduction: Melatonin is a hormone used in the treatment of diverse pathologies due to its ability to regulate numerous physiological processes related to biological rhythms and neuroendocrine function.

Material and methods: This study examines whether or not the antioxidant capacities of melatonin are modified during the creation of fast disintegrating oral tablets (FDDTs) through direct compression in which different concentrations of the active substance $(3,5,10$ and $60 \mathrm{mg}$ ) and excipients are made. In addition, the antioxidant capacity of melatonin is compared with that of reference antioxidants such as vitamin C, Trolox, resveratrol, glutathione and nicotinamide adenine dinucleotide phosphate (NADPH).

Results: Melatonin has a lower antioxidant capacity only $5 \%$ of the capacity of resveratrol. Resveratrol is the compound having the greatest antioxidant capacity. As for the influence of the tablets components, it was found that only at higher concentrations of melatonin $(60 \mathrm{mg} / \mathrm{tablet})$, with the excipients mannitol, polyvinylpyrrolidone (crospovidone), magnesium stearate and anhydrous colloidal silica, did a decrease occur in the antioxidant capacity value, possibly due to the lower percentage of these excipients in the formulation.

Key words: melatonin, antioxidant, total antioxidative capacity, ferric ion reducing antioxidant power, fast dissolving/disintegrating tablets.

\section{Introduction}

Melatonin (MT) is a hormone derived from 5-hydroxytryptamine, secreted mainly by the pineal gland and vertebrate retina during periods of darkness [1]. Its importance lies in its ability to regulate numerous physiological processes related to biological rhythms and neuroendocrine function [2].

Melatonin has been extensively cited as a powerful antioxidant, both in vitro and in vivo. A large part of its effectiveness in vivo may be attributed to the cascade of melatonin antioxidant metabolites that are produced. Unlike most small-molecule biological antioxidants such as vitamin C (ascorbic acid), $\alpha$-tocopherol (vitamin E), lipoic acid, etc., melatonin does not undergo a redox cycle. It undergoes molecular rearrangement, effectively removing the free electron from the system - a so-called suicidal antioxidant (Figure 1). Each of the products of the rearrangement is also

\author{
Corresponding author: \\ Adolfina Ruiz PhD \\ Pharmacy and \\ Pharmaceutical Technology \\ Department \\ School of Pharmacy \\ University of Granada \\ 18071 Granada, Spain \\ Phone: +34 58243904 \\ Fax: +34 58248958 \\ E-mail: adolfina@ugr.es
}


Antioxidant cascade

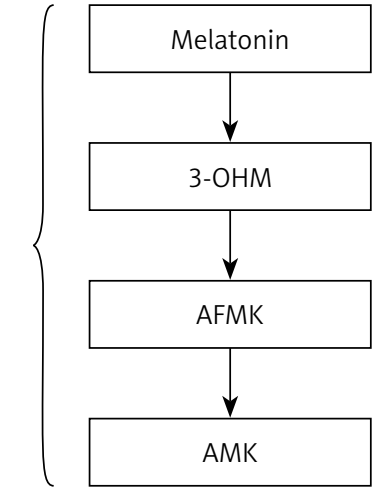

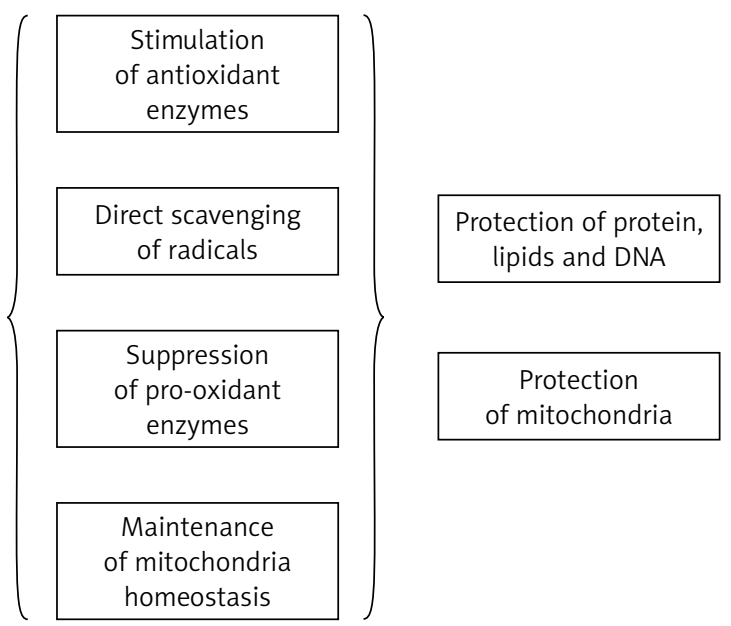

Figure 1. Melatonin's antioxidant cascade and mechanisms of protection against oxidative damage. The protection of mitochondria by melatonin and its metabolites is considered as an independent mechanism of cellular protection. However, the other three mechanisms may all be involved in maintaining mitochondrial homeostasis

a potent antioxidant in its own right. Furthermore, most of these processes involve more than one reactive oxygen species (ROS) per step; thus one melatonin molecule could potentially scavenge up to 10 radical species before the final metabolite is eliminated from the body [3].

Mechanistically speaking, melatonin may stimulate ROS production through its interaction with calmodulin. It may also interact with mitochondrial complex III or the mitochondrial transition pore to promote ROS production (Figure 1) [4].

It has been demonstrated that melatonin is capable of inhibiting oxidative damage caused by neurotransmitters such as kainic acid or glutamate in cerebellar granules or in the brain $[5,6]$, and excitotoxic damage of $\mathrm{N}$-methyl-D-aspartate (NMDA) in the striate cortex and of NMDA [7] and hypoxia/reoxygenation of the primary cultures of cortical neurons in rats [8]. Based on these actions, many neurodegenerative pathologies associated with the aging process, such as Alzheimer's and Parkinson's disease, epilepsy, and processes of ischemia-reperfusion [9], may be improved through the use of antioxidants such as melatonin, since these phenomena involve the production of free radicals [10].

On the other hand, diverse enzymes having antioxidant activity may be regulated by melatonin, as is the case with glucose-6-phosphate dehydrogenase (hepatic and cerebral) and the activity of glutathione peroxidase. Both show increased activity at pharmaceutical dosages [11].

In this study, the in vitro antioxidant capacity (AC) of melatonin was determined using the total antioxidant capacity (TAC) method, in which scavenging capacity was determined and compared with the antioxidant capacity of other compounds that are present, some of which are very commonly used in pharmaceuticals, such as res- veratrol, vitamin C (sodium ascorbyl phosphate [Na P-ascorbyl]), Trolox (a water-soluble vitamin $\mathrm{E}$ analog), glutathione and nicotinamide adenine dinucleotide phosphate (NADPH). In the second phase, the influence of the excipients and preparation technique on the $A C$ was determined based on the use of different formulations of oral fast dissolving/disintegrating tablets (FDDTs).

\section{Material and methods}

\section{Chemicals}

Melatonin was purchased from Methapharmaceutical IND S.L. (Spain). Mannitol, polyvinylpyrrolidone (crospovidone), lactose, magnesium stearate, anhydrous colloidal silica, tartaric acid and sodium bicarbonate were all supplied by Fagron Ibérica S.A.U. (Spain).

The antioxidants, vitamin C (sodium ascorbyl phosphate) and resveratrol were supplied by Fagron Ibérica S.A.U. (Spain). NADPH, adenosine triphosphate (ATP) and glutathione (GSH) were purchased from Sigma Aldrich (Spain).

For the determination of total antioxidant activity using the ferric ion reducing antioxidant power (FRAP) technique, the following were used: sodium acetate, acetic acid, 2,4,6-tris(2-pyridyl)-striazine (TPTZ), hydrochloric acid, ferric chloride, $\mathrm{FeSO}_{4} \times 7 \mathrm{H}_{2} \mathrm{O}$ and bidistilled water, purchased from Sigma Aldrich (Spain).

For the determination of total antioxidant activity using the TAC technique, the following were used: sodium acetate, bidistilled water, glacial acetic acid, hydrogen peroxide, 2,2'-azino-bis-2-ethybenzothiazoline-6-sulphonic acid (ABTS), the water-soluble analogue of vitamin E (Trolox (6-hydroxy-2,5,7,8-tetramethylchroman-2-carboxylic acid)), ethanol, methanol and monosodium phosphate, purchased from Sigma Aldrich (Spain). 


\section{Methods}

For the measurement of the different optical densities, an 8500 UV/VIS Dynco spectrophotometer (Denmark) was used.

For the manufacturing of the orodispersible melatonin tablets (FDDTs) a Specac pellet press was used (Atlas Series TM (Germany)), as well as a manual $15 \mathrm{t}$ press and 5 and $10 \mathrm{~mm}$ diameter pellet dies.

\section{Ferric ion reducing antioxidant power (FRAP)}

This method is used to indirectly determine antioxidant capacity. It is based on the power of an antioxidant substance to reduce $\mathrm{Fe}^{3+}$ to $\mathrm{Fe}^{2+}$, which has fewer antioxidant properties. The colorless ferric compound TPTZ is reduced to a colored ferric compound $[12,13]$. The reagents created for the technique are mixed in an $\mathrm{A}: \mathrm{B}: \mathrm{C}(10: 1: 1)$ relation. Reagent $A$ is made with $300 \mathrm{mM}$ sodium acetate in water and $1.6 \%$ acetic acid ( $\mathrm{pH}$ 3.6). Reagent $B$ is a ferric compound, TPTZ $10 \mathrm{mM}$ in $40 \mathrm{mM}$ of $\mathrm{HCl}$ in water, and finally, reagent $\mathrm{C}$ is made up of $\mathrm{FeCl}_{3}, 20 \mathrm{mM}$, in water. The reader is tempered at $37^{\circ} \mathrm{C}$ for $5 \mathrm{~min}$. A $250 \mu \mathrm{l}$ mixture of the previously described reagents is added and the first absorbance measurement is made at $593 \mathrm{~nm}$, serving as initial absorbance data. Next, $40 \mu \mathrm{l}$ of melatonin is added in triplicate, conveniently diluted in a water-alcohol solution (1: 1.5; ethanol: $\mathrm{pH} 7$ buffer), as well as the $\mathrm{FeSO}_{4}$ standard, in order to calibrate. As a blank, $40 \mu$ l of bidistilled water is used. The cuvette is introduced into the reader. It is agitated and after $4 \mathrm{~min}$, the reaction takes place. Finally, absorption is measured at
$593 \mathrm{~nm}$ in order to obtain the final absorbance values.

\section{TAC: ABTS $^{*+}$ generation and determination of antioxidant capacity}

The method developed by Erel 2004 [14] was applied. The antioxidant capacity of the samples was measured based on the capacity of the same to capture the free radicals present in the medium. This method consists of ABTS, oxidized to ABTS *+ in the presence of hydrogen peroxide (final concentration, $2 \mathrm{mM}$ ) in an acidic medium (acetate buffer $30 \mathrm{mM}, \mathrm{pH}$ 3.6). In this medium, the ABTS concentrate $(10 \mathrm{mM})$ has a dark green color and is more stable. When the ABTS ${ }^{*+}$ is diluted to a final concentration of $1.3 \mathrm{mM}$ in a buffered solution with a higher $\mathrm{pH}$ (acetate buffer $0.4 \mathrm{M}, \mathrm{pH}$ 5.8) the characteristic dark green color of ABTS*+ disappears and it slowly whitens over time. The antioxidants studied in this process accelerate the whitening in a manner that is proportional to their concentration. This reaction may be monitored spectrophotometrically and the rate of disappearance of $\mathrm{ABTS}^{\cdot+}$ (whitening) may be inversely related to the overall antioxidant capacity of the tested substances.

\section{Preparation of FDDTs}

Tables I and II show the composition of the tested formulations. They are divided into two groups based on the difference in excipients used during manufacturing, process development and subsequent Galenic tests based on the work of Muñoz et al. $[2,15]$.

Table I. Fast dissolving disintegrating tablets obtained by direct compression

\begin{tabular}{|lccccc|}
\hline Formulation & $\begin{array}{c}\text { Melatonin } \\
(\%)\end{array}$ & $\begin{array}{c}\text { Mannitol } \\
(\%)\end{array}$ & $\begin{array}{c}\text { Polyvinylpyrroli- } \\
\text { done (\%) }\end{array}$ & $\begin{array}{c}\text { Magnesium } \\
\text { stearate (\%) }\end{array}$ & $\begin{array}{c}\text { Anhydrous collo- } \\
\text { idal silica (\%) }\end{array}$ \\
\hline 1 & 2 & 66.67 & 28 & 2 & 1.33 \\
\hline 2 & 3.33 & 66.67 & 26.67 & 2 & 1.33 \\
\hline 3 & 6.67 & 63.33 & 26.67 & 1.33 & 0.66 \\
\hline 4 & 40 & 44.67 & 13.33 & 2 & 28 \\
\hline
\end{tabular}

Table II. Fast dissolving disintegrating tablets obtained by direct compression, with effervescents' disintegration

\begin{tabular}{|lccccc|}
\hline Formulation & $\begin{array}{c}\text { Melatonin } \\
(\%)\end{array}$ & $\begin{array}{c}\text { Tartaric acid } \\
(\%)\end{array}$ & $\begin{array}{c}\text { Sodium } \\
\text { bicarbonate (\%) }\end{array}$ & $\begin{array}{c}\text { Polyvinylpyrroli- } \\
\text { done (\%) }\end{array}$ & $\begin{array}{c}\text { Anhydrous collo- } \\
\text { idal silica (\%) }\end{array}$ \\
\hline 5 & 2 & 23.33 & 31.33 & 42 & 1.33 \\
\hline 6 & 3.33 & 23.33 & 31.33 & 40.67 & 40 \\
\hline 7 & 6.67 & 22 & 30 & 20 & 0.33 \\
\hline 8 & 35.29 & 17.65 & 32.67 & 42 & 0.67 \\
\hline Blank & - & 23.33 & 31.33 & 4.33 \\
\hline
\end{tabular}




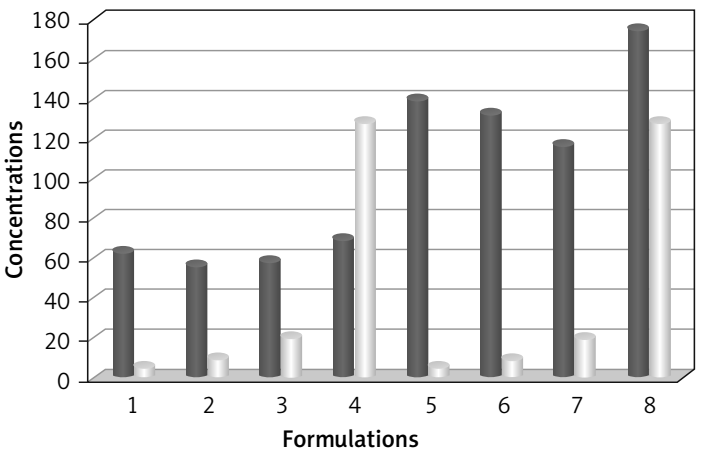

- Theoretical concentration $\quad \square$ Real concentration

Figure 2. Results of the concentrations obtained with the FRAP technique

\section{Results and discussion}

First, the antioxidant capacity curves for melatonin are made based on the TAC and FRAP methods (Figures 2 and 3), using a measurement range between 0 and $50 \mathrm{mM}$ of the active substance, obtaining straight lines for the pattern when facing the $\mathrm{mM}$ of melatonin with respect to the increase in absorbance (optical density), in order to compare the results of the antioxidant capacity of the tablets. Pastoriza et al. [16] determined the antioxidant capacity by different in vitro methodologies (ABTS, FRAP, DPPH, ORAC, HOSC) in the

Table III. Theoretical concentrations of the different formulations

\begin{tabular}{|lc|}
\hline Formulations & Theoretical concentrations $[\mathrm{mM}]$ \\
\hline 1 & 6.46 \\
\hline 2 & 10.77 \\
\hline 3 & 21.53 \\
\hline 4 & 129.20 \\
\hline 5 & 6.46 \\
\hline 6 & 10.77 \\
\hline 7 & 21.53 \\
\hline 8 & 129.20 \\
\hline
\end{tabular}

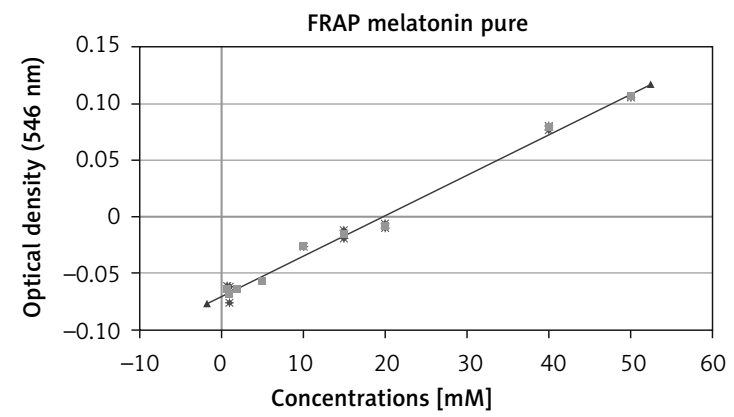

Figure 4. FRAP. Ferric ion reducing antioxidant power $\left(R^{2}=0.9934\right.$, error $\left.=0.004905\right)$

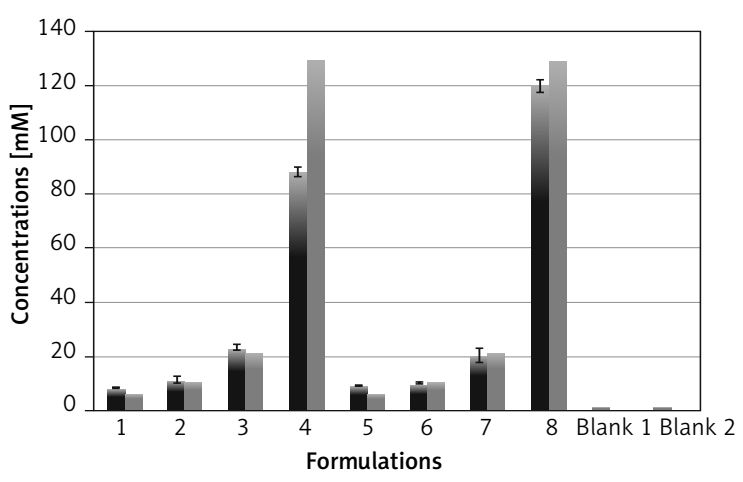

- Real concentration Theoretical concentration

Figure 3. Results of the concentrations obtained with the TAC technique

Spanish diet. They concluded that the ABTS method is one of the most often used in the scientific literature and adequate, cause this method is based on the electron transference.

For the dissolution of the pure melatonin and for the content in the FDDTs, a mixture of the potassium phosphate buffer at $\mathrm{pH} 7$ and ethanol was used at a ratio of $1: 1$. After conducting various dissolution tests, it was found that for formulations 4 and 8, only ethanol should be used as a solvent. After completely dissolving the melatonin, it is centrifuged at 10,000 rpm for $5 \mathrm{~min}$ in an Eppendorf 5804 centrifuge and the measurement of optical density is conducted via spectrophotometry.

The absorbance values of $\mathrm{ABTS}^{\cdot+}$ at a wavelength of $546 \mathrm{~nm}$ reveal a good correlation with the ABTS $^{*+}$ concentration when preparing a curve pattern with an interval concentration of $1.3-50 \mu \mathrm{M}$. The correlation between the absorbance values of ABTS $^{*+}$ and the concentration was linear.

In Table III, the theoretical concentrations are presented for the different tablets that were studied (data obtained in the extrapolation of data from the antioxidant capacity curves).

Figure 4 reveals the calibration of the melatonin obtained based on the FRAP method $\left(R^{2}=\right.$ 0.9934 , error $=0.004905)$, and Figure 5 show the calibration based on TAC method $\left(R^{2}=0.9964\right.$, error $=0.005831)$.

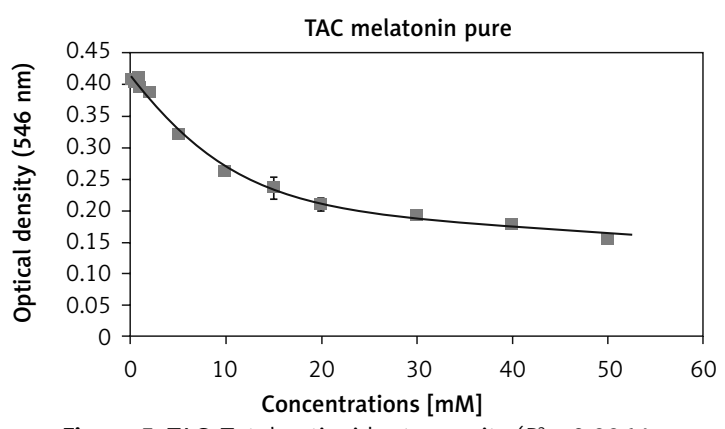

Figure 5. TAC. Total antioxidant capacity $\left(R^{2}=0.9964\right.$, error $=0.005831$ ) 
A

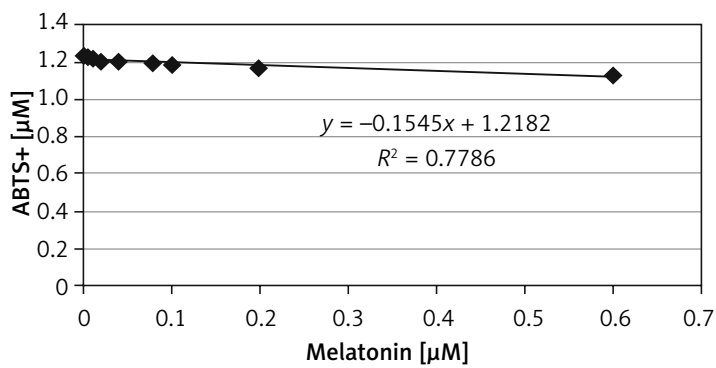

C

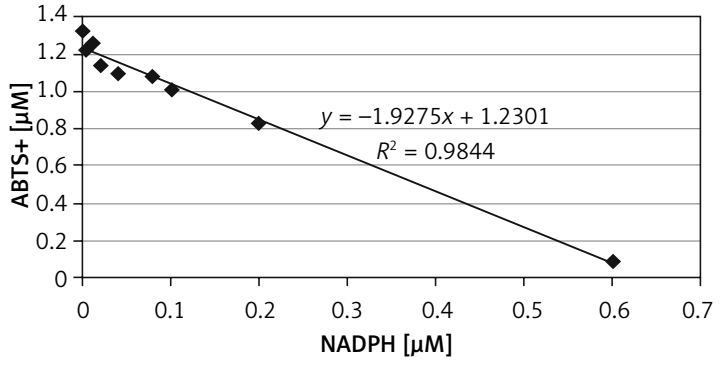

E

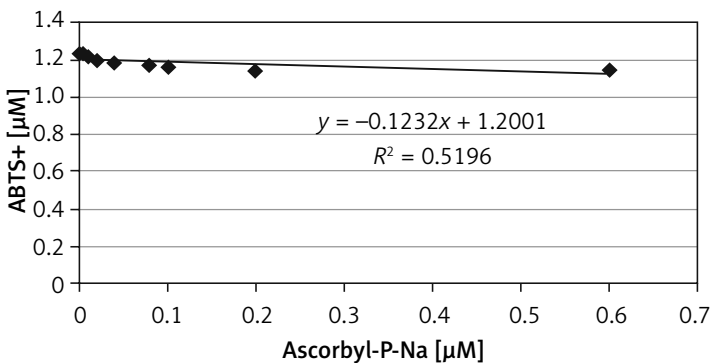

B

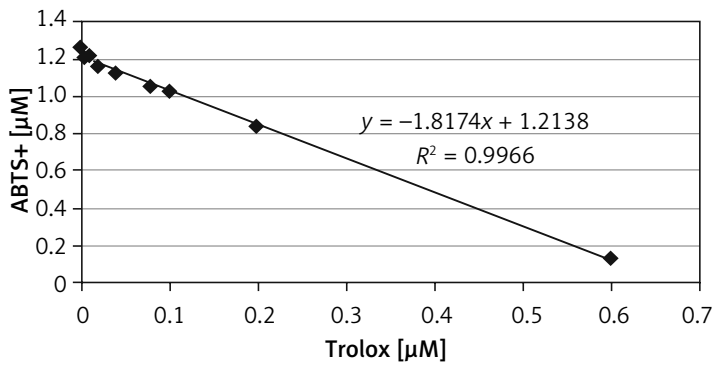

D

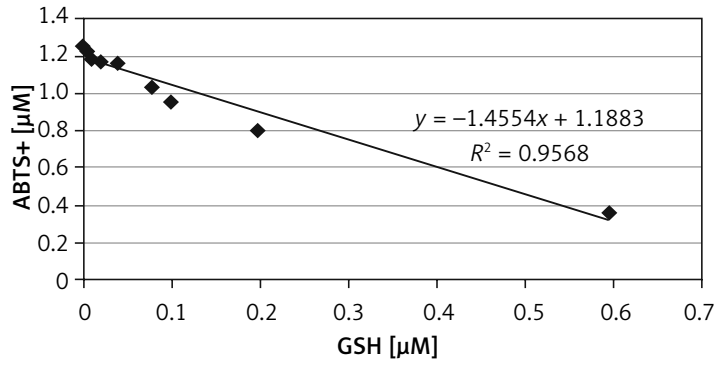

$\mathrm{F}$

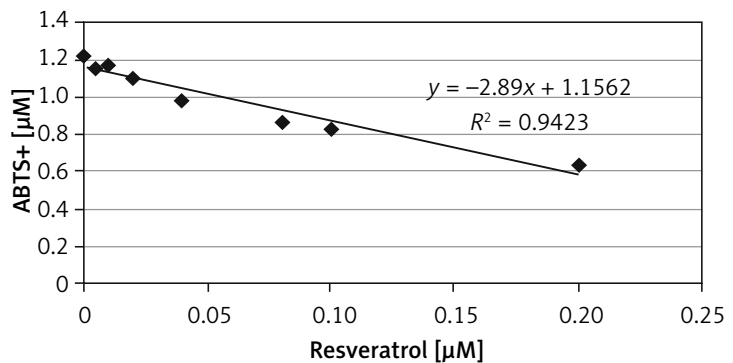

Figure 6. Stoichiometric analysis of different antioxidants tested

Once the calibration curve is obtained and the samples are prepared, the antioxidant capacity of the different formulations of melatonin was initially determined based on two methods (FRAP and TEAC). The FRAP method was discarded since it was found that excipients of the different formulations interacted with the reagents of the method, whereby with greater doses of melatonin (formulation 4 and 8), having lower proportional quantities of excipients, there was a lower turbidity produced upon adding the reagents and the obtained values were closer to the theoretical values (Figure 4).

\section{$A C$ in the reference antioxidants}

For the comparison of antioxidant capacity between the different tested compounds, a water-alcohol solution was used as a solvent for MT and Trolox (1 : 1.5; ethanol: pH 7 buffer); in the case of NADPH, GSH and sodium ascorbyl phosphate, a buffer of $\mathrm{pH} 7$ was used and for resveratrol, ethanol was used.

Figures 6 and 7 reveal the results obtained for the different antioxidant substances.
The antioxidant capacity of melatonin was tested, as well as that of other synthetic substances having well-known antioxidant roles in humans, such as vitamin C, NADPH, GSH, Trolox (analogue of vitamin $\mathrm{E}$ ) and a plant phytoalexin, resveratrol. All of the compounds were found to have an antioxidant capacity, as disappearance of ABTS*+ was found and this disappearance was dependent on the antioxidant concentration.

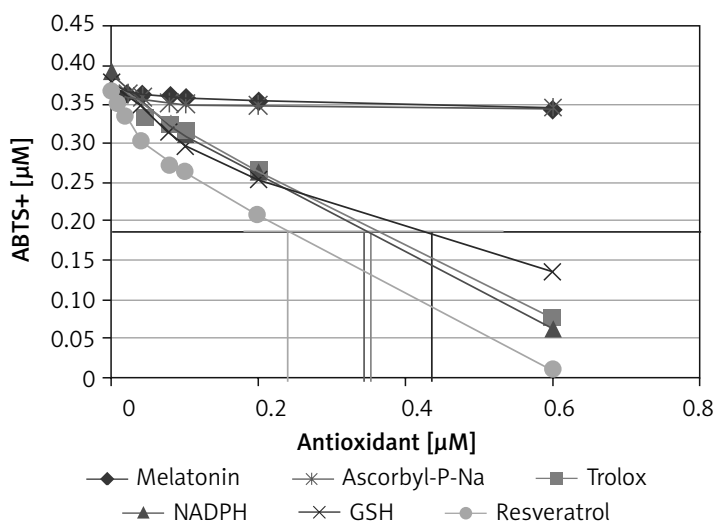

Figure 7. Comparison of the antioxidant capacity of different substances 
The greatest antioxidant activity was found in resveratrol (Figure 6 F), with NADPH and Trolox (Figures 6 B, C) having quite similar capacities, at 66 and $62 \%$ of the capacity found in resveratrol. GSH (Figure 6 D) was found to have approximately $50 \%$ of the antioxidant capacity of resveratrol. Surprisingly, the antioxidant values of melatonin (Figure $6 \mathrm{~A}$ ) and ascorbyl-P-Na (Figure $6 \mathrm{E}$ ) were notably lower than those found for the other substances, only $5 \%$ and $4 \%$ respectively of the activity of resveratrol.

Resveratrol is a plant derivative, a phytoalexin of the Polygonum cuspidatum root, having quite interesting properties and applications, despite the fact that few clinical trials have been carried out on it. It has anti-inflammatory, anti-thrombogenic, anti-tumor, anti-osteoporosis, immunestimulating and antioxidant effects, and its usefulness has been demonstrated in the treatment of skin ageing [17]. Glutathione is one of the most important intracellular antioxidants thanks to its thiol group $(-\mathrm{SH})$, which is an effective antioxidant. NADPH is another well-known intracellular antioxidant, thanks to its capacity to donate $\mathrm{H}^{+}$, enabling it to neutralize a large variety of free radicals [18]. On the other hand, vitamins are also recognized antioxidants. Detaining the reactive cascade of the free radicals, these vitamins, such as vitamins $C$ and $E$, are obtained through the diet. Finally, melatonin has been frequently described as a hormone offering numerous beneficial effects on the organism and many treatment possibilities for different illnesses, particularly those resulting from an imbalance of the cellular redox state; thus its antioxidant properties are the most well known of its benefits. Being a hormone, its in vivo effect may differ from its in vitro effect, given that the

Table IV. Results from real concentrations after extrapolation of the data in the calibration curve

\begin{tabular}{|lccc|}
\hline Formulations & $\begin{array}{c}\text { Real } \\
\text { concentra- } \\
\text { tion [nM] }\end{array}$ & Error & $\begin{array}{c}\text { Theoretical } \\
\text { concentra- } \\
\text { tion [nM] }\end{array}$ \\
\hline 1 & 8.8222 & 0.2971 & 6.46 \\
\hline 2 & 11.7372 & 1.1466 & 10.77 \\
\hline 3 & 23.6120 & 1.0634 & 21.53 \\
\hline 5 & 88.3630 & 1.6740 & 129.20 \\
\hline 6 & 9.4037 & 0.2096 & 6.46 \\
\hline 7 & 10.3260 & 0.2906 & 10.77 \\
\hline 8 & 21.0307 & 2.4327 & 21.53 \\
\hline Blank 1 & 120.4130 & 1.9287 & 129.20 \\
\hline Blank 2 & 1.6381 & - & - \\
\hline
\end{tabular}

target cells may increase its antioxidant potential, detaining the cascade of free radicals.

The stoichiometric analysis indicates the need for approximately one molecule of NADPH, Trolox or GSH in order to reduce two molecules of ABTS ${ }^{*+}$. One molecule of resveratrol is needed to reduce 3.5 molecules of ABTS ${ }^{*+}$ and 6 molecules of melatonin and 7.5 molecules of ascorbyl-P-Na are necessary to reduce one molecule of $\mathrm{ABTS}^{*}$. These data were revealed from the $I C_{50}$ values (antioxidant concentration required in order to inhibit the reaction by $50 \%)$ for resveratrol $(0.18 \mu \mathrm{M})$; NADPH $(0.31 \mu \mathrm{M})$; Trolox $(0.32 \mu \mathrm{M})$; GSH $(0.38 \mu \mathrm{M})$; melatonin $(3.89 \mu \mathrm{M})$ and ascorbyl-P-Na $(4.65 \mu \mathrm{M})$ in scavenging $1.3 \mu \mathrm{M} \mathrm{ABTS}{ }^{*+}$ (Figure 7 ).

The differences in the antioxidant profile of the measured substances differ from one another. One molecule of NADPH, Trolox and GSH is capable of reducing two molecules of $\mathrm{ABTS}^{*+}$. The antioxidant mechanism of these molecules is based on the donation of $\mathrm{H}^{+}$. In order to perceive the antioxidant capacity of melatonin, it is necessary to use higher concentrations, as occurs with the higher doses administered in the treatment of Duchenne muscular dystrophy [2]. Sodium ascorbyl phosphate is a more stable formula of vitamin C, but perhaps the chemical stability attained in the formula is not compatible with the elimination of free radicals based on the described method. Finally, resveratrol is capable of producing the whitening of 3.5 molecules of ABTS ${ }^{\bullet}$, wherein the presence of benzene rings and double bonds permits the elimination of free radicals in a greater proportion than that which has been observed in other molecules [19].

\section{Determination of AC in FDDTs}

In the results obtained (Figure 3 and Table IV) we see that formulations $1,2,3,5,6,7$ and 8 maintain the antioxidant capacity detected for melatonin after the different galenic processes of its creation. In contrast, formulation 4, having a greater dosage of MT, is the only one presenting a decrease in the antioxidant capacity of the dosed melatonin, perhaps due to the fact that, compared to the other formulations, it has a lower proportion of excipients that may protect the active substance from the other technological processes and that, therefore, make it more vulnerable to degradation and antioxidant capacity.

If we compare the two groups of formulas (different excipients), we see (Figure 3 ) that there is a lower loss of antioxidant capacity in those that contain effervescent excipients, since the data indicate an increased proximity of the concentrations obtained after the trial of the different formulations to the theoretical concentrations (Table IV). This improvement in the conservation of the antioxidant activity of melatonin is more noticeable 
in the FDDTs with greater active substance concentrations (formulation 8), since, as observed, the proximity of the theoretical concentration to the real concentration is much greater than that of formulation 4 , perhaps due to the protection offered by the effervescent excipients on the melatonin and the greater proportion contained in the $60 \mathrm{mg}$ formulation, as compared to formulation 4.

Upon analysis of the obtained data, it is proposed that higher melatonin concentrations are necessary. Patients should be administered the formulation with effervescent excipients, since, in this case, the melatonin maintains almost all of its antioxidant capacity.

In conclusion, the TAC method was used to determine $A C$ in the comparison of the reference antioxidant substances and for the AC trial on the different FDDTs of melatonin at distinct concentrations and with different excipients, after discarding the FRAP method due to interactions with the excipients of the different FDDTs.

Our results demonstrate that melatonin has a lower antioxidant capacity than resveratrol.

The different melatonin concentrations contained in the orodispersible tablets were found to maintain their AC, except in the case of the $60 \mathrm{mg}$ tablets (formulation 4). Therefore, for higher concentrations, it is recommended that effervescent tablets be used, as they do not modify the AC of MT.

\section{Conflict of interest}

The authors declare no conflict of interest.

\section{References}

1. Flórez J, Armijo JA. Neurotransmisión en el sistema nervioso central. In: Farmacología Humana. Florez J (ed.). Masson-Salvat Medicina, Barcelona 2008; 475-6.

2. Muñoz H, Castan H, Clares B, Ruiz MA. Obtaining fast dissolving disintegrating tablets with different doses of melatonin. Int J Pharm 2014; 467: 84-9.

3. Johns JR, Platts JA. Theoretical insight into the antioxidant properties of melatonin and derivatives. Platts Org Biomol Chem 2014; 12: 7820-66.

4. Zhang HM, Zhang Y. Melatonin: a well-documented antioxidant with conditional pro-oxidant actions. J Pineal Res 2014; 57: 131-46.

5. Aziz Ibrahim IA, Kamisah Y, Nafeeza MI, Nur Azlina MF. The effects of palm vitamin $E$ on stress hormone levels and gastric lesions in stress-induced rats. Arch Med Sci 2012; 8: 22-9.

6. Ahmed EA, Omar HM, Elghaffar SK, Ragb SM, Nasser AY. Food Chem Toxicol 2011; 49: 1115-21.

7. Skaper, SD, Ancona B, Facci L, Franceschini D, Giusti P. Melatonin prevents the delayed death of hippocampal neurons induced by enhanced excitatory neurotransmission and the nitridergic pathway. FASEB J 1998; 12: 725-31.

8. Cazevieille C, Safa R, Osborne NN. Melatonin protects primary cultures of rat cortical neurons from NMDA ex- citotoxicity and hypoxia/reoxygenation. Brain Res 1997; 768: 120-4.

9. Abogresha NM, Greish SM, Abdelaziz EZ, Khalil WF. Remote effect of kidney ischemia-reperfusion injury on pancreas: role of oxidative stress and mitochondria lapoptosis. Arch Med Sci 2016; 12: 252-62.

10. Tan CH, Chen LD, Poeggeler B, Manchester LC, Reiter RJ. Melatonin: a potent, endogenous hydroxyl radical scavenger. Endocrine J 1993; 1: 57-60.

11. Barlow-Walden LR, Reiter RJ, Abe M, Pablos MI, Menéndez-Peláez A, Chen LD, Poeggler B. Melatonin stimulates brain gluthatione peroxidase activity. Neurochem Int 1995; 26: 497-502.

12. Miller NJ, Rice-Evans C, Davies MJ, Gopinathan V, Milner A. A novel method for measuring antioxidant capacity and its application to monitoring the antioxidant statusin premature nenates. Clin Sci (Lond) 1993; 84: 407-12.

13. Pulido R, Bravo L, Saura-Calisto F. Antioxidant activity of dietary polyphenols as determined by a modifiedferric reducing/antioxidant power assay. J Agric Food Chem 2000; 48: 3396-402.

14. Erel O. A novel automated direct measurement method for total antioxidant capacity using a new generation, more stable ABTS radical cation. Clin Biochem 2004; 37: 277-85.

15. Ruiz MA, Muñoz H. Composición bucodispersable de melatonina. ES Patent 2013; P201231477.

16. Pastoriza S, Rufian-Henares JA. Contribution of melanoidins to the antioxidant capacity of the Spanish diet. Food Chem 2014; 164: 438-45.

17. Sweetman S. Guía complete de consultas farmacoterapéuticas. Vol. 1. $6^{\text {th }}$ ed. Martindale Pharma, Barcelona (Spain) 2011; 3552.

18. Kirsch $M$, Groot $H$. NAD(P)H, a directly operating antioxidant? FASEB J 2001; 15: 1569-74.

19. Akinci A, Esrefoglu M, Cetin A, Ates B. Melatonin is more effective than ascorbic acid and beta-carotene in improvement of gastric mucosal damage induced by intensive stress. Arch Med Sci 2015; 11: 1129-36. 